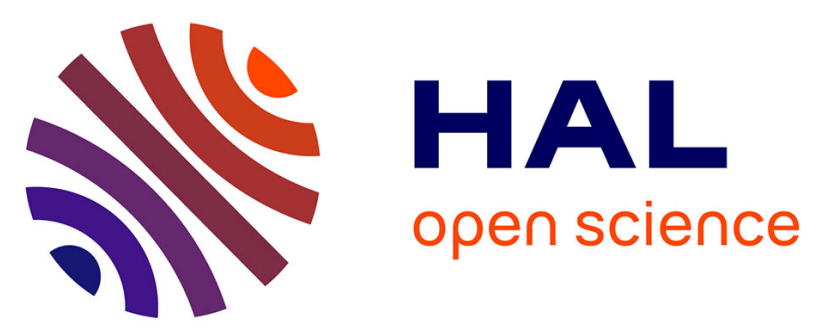

\title{
ROBUST FOVEAL AVASCULAR ZONE SEGMENTATION AND ANATOMICAL FEATURE EXTRACTION FROM OCT-A HANDLING INTER-EXPERT VARIABILITY
}

Charles Jabour, Damien Garcia, Thibaud Mathis, Olivier Loria, Cédric Rochepeau, Brahim Harbaoui, Pierre Lantelme, Didier Vray, Odyssée Merveille

\section{- To cite this version:}

Charles Jabour, Damien Garcia, Thibaud Mathis, Olivier Loria, Cédric Rochepeau, et al.. ROBUST FOVEAL AVASCULAR ZONE SEGMENTATION AND ANATOMICAL FEATURE EXTRACTION FROM OCT-A HANDLING INTER-EXPERT VARIABILITY. International Symposium on Biomedical Imaging, Apr 2021, Nice (virtual), France. hal-03154386

\author{
HAL Id: hal-03154386 \\ https://hal.science/hal-03154386
}

Submitted on 28 Feb 2021

HAL is a multi-disciplinary open access archive for the deposit and dissemination of scientific research documents, whether they are published or not. The documents may come from teaching and research institutions in France or abroad, or from public or private research centers.
L'archive ouverte pluridisciplinaire HAL, est destinée au dépôt et à la diffusion de documents scientifiques de niveau recherche, publiés ou non, émanant des établissements d'enseignement et de recherche français ou étrangers, des laboratoires publics ou privés. 


\title{
ROBUST FOVEAL AVASCULAR ZONE SEGMENTATION AND ANATOMICAL FEATURE EXTRACTION FROM OCT-A HANDLING INTER-EXPERT VARIABILITY
}

\author{
Charles Jabour ${ }^{\star}$, Damien Garcia ${ }^{\star}$, Thibaud Mathis ${ }^{\dagger}$, Olivier Loria ${ }^{\dagger}$, \\ Cédric Rochepeau ${ }^{\dagger \dagger}$, * Brahim Harbaoui ${ }^{\ddagger \star}$, Pierre Lantelme ${ }^{\ddagger \star}$, Didier Vray ${ }^{\star}$, Odyssée Merveille ${ }^{\star}$ \\ * Univ Lyon, INSA-Lyon, Université Claude Bernard Lyon 1, UJM-Saint Etienne, CNRS, Inserm, \\ CREATIS UMR 5220, U1206, F-69100, Lyon, France \\ ${ }^{\dagger}$ Service d'Ophtalmologie, Hôpital Universitaire de la Croix-Rousse, Hospices Civils de Lyon, \\ UMR-CNRS 5510 MATEIS, Université Lyon 1, Lyon, France \\ ${ }^{\ddagger}$ Croix Rousse University Hospital, Cardiology Department, Hospices Civils de Lyon, Lyon, France \\ ${ }^{\dagger \dagger}$ Service d'Ophtalmologie, Hôpital Edouard Herriot, Hospices Civils de Lyon, Lyon, France
}

\begin{abstract}
The Foveal Avascular Zone (FAZ) is commonly analyzed from OCT-A images to diagnose retinal diseases. When quantitative measures are required, ophthalmologists manually draw the contours of the FAZ and compute several anatomical features from these annotations. However, there is no clear consensus among experts on the FAZ definition in pathological cases. In this work, we developed a novel framework to automatically segment the FAZ based on three different expert annotations to be robust to interexpert variability. A loss based on the Hausdorff Distance was used to specifically address complex pathological FAZ that are usually poorly segmented by automatic methods. We used a dataset of more than 200 images to train and test our model, and we achieved similar or even better segmentation performance than individual experts.
\end{abstract}

Index Terms - Foveal Avascular Zone, segmentation, OCT-A, inter-expert variability

\section{INTRODUCTION}

Optical Coherence Tomography Angiography (OCT-A) is a 3D non-invasive imaging method that detects blood flow in the eye fundus. OCT-A was successfully used to assess several vascular pathology such as diabetic retinopathy [1] or glaucoma [2]. The retinal vascular network is organized around the so-called Foveal Avascular Zone (FAZ). Most of the time, the FAZ appears as a dark circular central region on OCT-A images (see Figure 1 (a)). However, pathological subjects present a FAZ that may be crossed by vessels (see Figure 1 (b)) or be so small it cannot be differentiated from any other non-perfused area. In these cases, there is no clear consensus among ophthalmologists on the FAZ definition (see Figure 2). FAZ detection plays a key role in the diagnosis of several diseases. Its size, shape and surrounding perfusion are classic markers of diabetic retinopathy. It is therefore clinically relevant to provide a robust and effective method for detecting FAZ.

Segmentation of the FAZ is a problem often handled manually [3] [4]. However, this solution is timeconsuming and the results depend heavily on the expert.

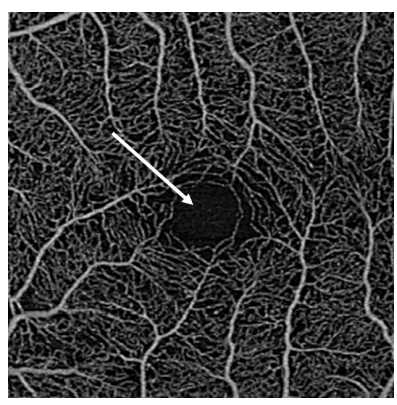

(a) Healthy FAZ

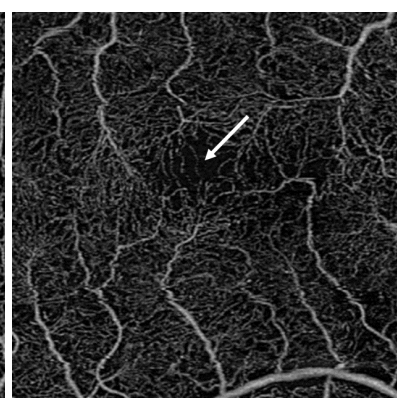

(b) Pathological FAZ
Fig. 1: Examples of the superficial retinal layer imaged by OCT-A. White arrows point to the Foveal Avascular Zone (FAZ). As shown Figure 2, pathological FAZ can be seen differently from one expert to another

To solve these issues, several approaches have been introduced. Diaz et al. [5] proposed to detect the FAZ as the widest dark connected component by applying morphological operations. Lu et al. [6] assumed the FAZ to be at the center of the image and used a region growing approach followed by an active contours algorithm. Nonetheless, these methods are parametric and are not designed to detect complex FAZ. Recently, deep learning methods based on UNet-like architectures were developed to automatically segment the FAZ from OCT-A [7, 8]. However, they did not take into account the variability among experts, which makes these approaches dependent on a single expert opinion. Heisler et al. [9] proposed another technique to detect the FAZ from a segmentation of the vascular network produced by U-Net. This approach assumes the vascular network to be fully connected, which is physiologically incorrect in most situations.

In this work, we proposed an automatic deep learning approach to detect the FAZ on OCT-A images. Unlike most state-of-the-art methods, our approach is able to detect healthy and complex non-healthy FAZ, and includes interexpert variability to provide robust results even in the most difficult cases. Our contribution is three-fold: 1) we built an annotated dataset relying on manual FAZ segmentation from three different experts and including complex cases, such as crossing vessels and absence of detectable FAZ, 


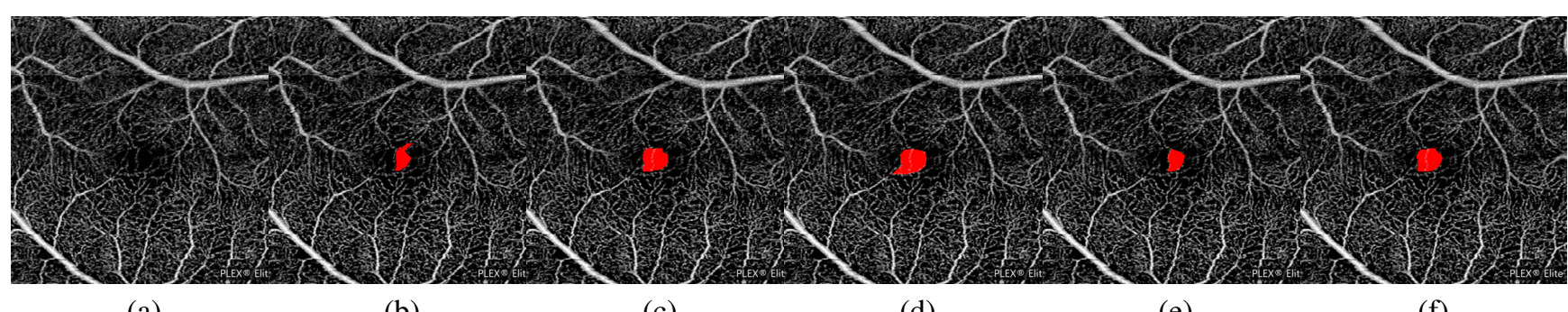

(a)

(b)

(c)

(d)

(e)

(f)

Fig. 2: Inter-expert variability on a complex FAZ. (a) original image; (b-e) annotations of the FAZ in red from 3 experts (one of them suggesting 2 possible annotations of the FAZ); (f) mean ground truth.

2) we used a loss based on the Hausdorff Distance (HD) to help the network detect FAZ with complex non-circular shapes, 3) we showed that FAZ markers extracted from our segmentations were in good agreement with those extracted from expert annotations.

\section{METHOD}

This section presents our approach to automatically detect the FAZ from OCT-A images and robustly extract features characterizing this FAZ. An illustration of the global approach is presented in Figure 3.

\subsection{Dataset}

In this work, we used a dataset composed of 204 OCT-A images of the superficial retinal layer acquired using a Zeiss Plex Elite 9000 swept source. The population included healthy patients, diabetic patients with different grades of diabetic retinopathy, and patients with coronary artery disease. Three experts delineated the FAZ in the 204 images. For each image, we adopted a majority voting policy to produce a unique ground-truth, called mean ground-truth from the different expert annotations. In particular, a pixel belonging to the FAZ in more than half of the expert annotations was considered a FAZ pixel in the mean ground truth, otherwise it was considered as a background pixel (see Figure 2). This mean ground-truth includes the inter-expert variability and provides a virtual consensual ground truth that was used to train our network.

We used a first-order spline interpolation to rescale the original $1024 \times 1024$ pixels images to $400 \times 400$ pixels to meet the network architecture requirements. We applied a gaussian filter prior to the interpolation to avoid aliasing artifacts. We also performed an histogram equalization followed by a normalization between 0 and 1 to reduce the intensity variability of the input images

\subsection{Network architecture}

We used a light UNet [7] architecture to detect the FAZ, as it had already given interesting results in FAZ detection. Light UNet is a version of the classic UNet architecture with fewer convolutional layers, thereby reducing training time. More details about the network architecture can be found in [7].

The original light UNet loss is a classic Binary Cross Entropy (BCE). In this work we modified this loss to better take into account the FAZ geometry, which is crucial to extract robust FAZ characteristics. We proposed to add an Hausdorff Distance (HD) loss, $L_{\mathrm{HD}}$, to the BCE loss. The HD measures the longest distance between two sets (in our case, the mean ground truth and the network prediction). Karimi et al. [10] proposed several implementations of the HD loss and we chose the morphological approach for its fast and robust estimation. Let $\mathbb{R}^{\Omega}$ be the set of real-valued $2 \mathrm{D}$ images of size $|\Omega|$ pixels, the HD loss of a prediction $I \in \mathbb{R}^{\Omega}$ is defined as follows:

$$
L_{\mathrm{HD}}\left(I_{\mathrm{gt}}, I\right)=\frac{1}{|\Omega|} \sum_{k=1}^{K} \sum_{\Omega}\left(\left(I_{\mathrm{gt}}-I\right)^{2} \ominus B\right) k^{\alpha}
$$

$I_{\mathrm{gt}} \in \mathbb{R}^{\Omega}$ is the mean ground truth, $\ominus_{k}$ is the erosion operator with structuring element $B, K \in \mathbb{N}$ is the maximum number of erosion applied, and $\alpha \in \mathbb{R}$ penalizes long distances between the ground truth and the prediction. We experimentally set $\alpha=1$.

The final loss was a weighted sum of the BCE and the HD loss (see Equation 2). We experimentally assessed the optimal weight value and fixed it to $\lambda=0.3$.

$$
L\left(I_{\mathrm{gt}}, I\right)=\operatorname{BCE}\left(I_{\mathrm{gt}}, I\right)+\lambda L_{\mathrm{HD}}\left(I_{\mathrm{gt}}, I\right)
$$

\subsection{Training strategy}

Among the 204 images of the dataset, 13 images presented a FAZ crossed by vessels. To increase the proportion of these complex FAZ in the dataset, we added a $90^{\circ}$ rotated version of each of these 13 images to the dataset. Our final dataset thus contained 217 images, and was split into a training set of 187 images and a testing set of 30 images.

The network was trained with an Adam optimizer, a batch size of 5 and an initial learning rate of $5 \times 10^{-4}$ with a decay of 0.02 when the validation loss reached a plateau during 5 epochs. An early stopping strategy was used when the validation loss did not decrease over 10 epochs.

As the number of complex FAZ images was limited, we performed a 5-fold cross validation to assess the robustness of our approach to the training dataset.

\section{RESULTS}

Each segmentation was compared to its mean ground truth and the following values were computed: true positives (TP), false positives (FP) and false negatives (FN). From 


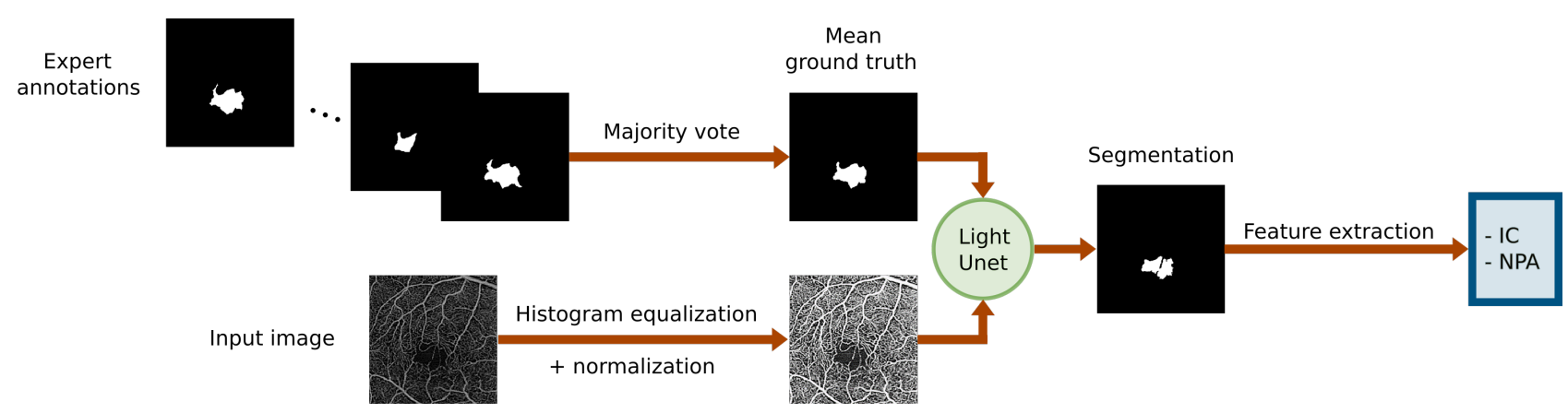

Fig. 3: Illustration of the global framework to extract anatomical FAZ features: Circularity Index (CI) and Non-perfused Area (NPA).

these values, we computed the Dice Similarity Coefficient (DSC) to evaluate the global segmentation quality (see Equation 3).

$$
D S C=\frac{2 \mathrm{TP}}{2 \mathrm{TP}+\mathrm{FP}+\mathrm{FN}}
$$

From a clinical point of view, achieving a good FAZ segmentation is not a goal in itself. Ophthalmologists use the FAZ segmentation to extract clinically relevant features characterizing the FAZ, such as the Circularity Index (CI) and the Non-Perfursed Area (NPA). The circularity index is defined as $\mathrm{CI}=4 \pi \frac{\mathrm{A}}{\mathrm{P}^{2}}$, where $\mathrm{A}$ and $\mathrm{P}$ are respectively the area and perimeter of the FAZ. The NPA is the inverse of the vessel density in an area of $300 \mu \mathrm{m}$ around the FAZ.

We applied the 5 models of the cross validation on our test set and obtained a mean DSC of $0.909 \pm 0.025$. This shows that our method is able to robustly provide good segmentation of the FAZ even on complex cases. We then selected the model with the lowest validation loss and compared its results to the expert manual segmentations. The quantitative results are presented in Table 1 . We conducted an ablative study to analyse the interest of adding the HD loss. The results shows that the HD loss both improved the DSC and the feature estimation errors. Indeed, we observed that the HD loss greatly improved the segmentation of complex FAZ (see Figure 4) which led to better global results.

To compare our results to the inter-expert variability, we confronted each expert annotation with the mean ground-truth and computed their DSC as well as the relative errors of the features (CI and NPA). Our approach was able to segment the FAZ with an accuracy similar to a single expert (the mean DSC was even greater and with less variability than one of the expert). Our estimation of the $\mathrm{CI}$ is generally in average less accurate than the one of the experts. The circularity index is indeed extremely dependent on small contour variations. Despite the great general segmentation performances that we obtained, small missing regions in some images may lead to high errors on the circularity index which explains these results. Finally, our network is able to estimate a more accurate NPA than those of most experts with a great robustness.

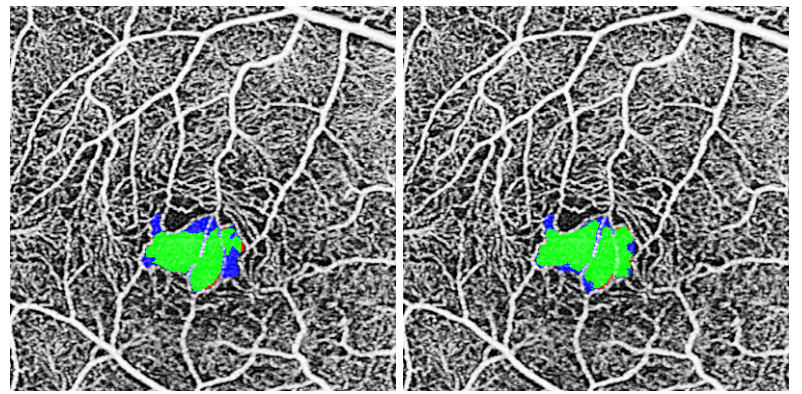

(a) without HD loss

(b) with HD loss

Fig. 4: Results from our FAZ segmentation approach on a FAZ crossed by vessels compared to the mean groundtruth (green: TP, red: FP, blue: FN).

\section{CONCLUSION}

We developed a novel framework to automatically segment the FAZ from OCT-A images and extract anatomical features relevant for retinal diagnosis purpose. Our deep learning segmentation method uses an Hausdorff distance loss and includes knowledge from several experts to provide a robust and accurate FAZ segmentation, even in the more complex cases, that reaches, and sometimes exceeds, the inter-expert variability. In the future, we plan to improve the CI estimation by including more complex cases in the database and add constraints in the network. In particular, we could add constraints on the contour of the segmentation to better take into account the sudden shape variations of the FAZ contour in complex cases.

\section{COMPLIANCE WITH ETHICAL STANDARDS}

This study was performed in line with the principles of the Declaration of Helsinki. Approval was granted by the Ethics Committee of the Hospices Civils de Lyon the $07 / 28 / 2018$.

\section{ACKNOWLEDGMENTS}

This work was part of the COREYE project [NCT03739073] funded by the Fédération Française de Cardiologie. We also thank Christelle Szanatek, Fatou Langevin and Fatma Zourane, who participated in the creation of the database. 


\begin{tabular}{l|c|c|c} 
& DSC & \% error CI & \% error NPA \\
\hline \hline without HD loss & $0.916 \pm 0.109$ & $19.237 \pm 26.254$ & $1.798 \pm 3.983$ \\
with HD loss & $0.928 \pm 0.059$ & $18.770 \pm 25.141$ & $1.6 \pm 3.217$ \\
\hline Expert 1 & $0.921 \pm 0.179$ & $13.975 \pm 17.798$ & $2.966 \pm 11.377$ \\
Expert 2 & $0.957 \pm 0.058$ & $7.549 \pm 6.070$ & $1.351 \pm 1.211$ \\
Expert 3 & $0.958 \pm 0.053$ & $12.082 \pm 17.565$ & $2.679 \pm 9.043$
\end{tabular}

Table 1: Quantitative results of our approach (two first lines) and the inter-expert variability (three last lines). The presented measures are the Dice coefficient (DSC), the circularity index (CI) and the non-perfused area (NPA).

\section{REFERENCES}

[1] Adriano Carnevali, Riccardo Sacconi, Eleonora Corbelli, et al., "Optical coherence tomography angiography analysis of retinal vascular plexuses and choriocapillaris in patients with type 1 diabetes without diabetic retinopathy," Acta Diabetologica, vol. 54, no. 7, pp. 695-702, 2017.

[2] Adeleh Yarmohammadi, Linda M. Zangwill, Alberto Diniz-Filho, et al., "Optical coherence tomography angiography vessel density in healthy, glaucoma suspect, and glaucoma eyes," Investigative Ophthalmology \& Visual Science, vol. 57, no. 9, pp. OCT451OCT459, 2016.

[3] Manuel Casselholmde Salles, Anders Kvanta, Urban Amrén, and David Epstein, "Optical coherence tomography angiography in central retinal vein occlusion: correlation between the foveal avascular zone and visual acuity," Investigative Ophthalmology Visual Science, vol. 57, no. 9, pp. OCT242-OCT246, 2016.

[4] Wasim A. Samara, Emil A. T. Say, Chloe T. L. Khoo, et al., "Correlation of foveal avascular zone size with foveal morphology in normal eyes using optical coherence tomography angiography," Retina, vol. 35, no. 11, pp. 2188-2195, 2015.

[5] Macarena Díaz, Jorge Novo, Paula Cutrín, et al., "Automatic segmentation of the foveal avascular zone in ophthalmological OCT-A images," PLOS ONE, vol. 14, no. 2, pp. 1-2, 2019.

[6] Yansha Lu, Joseph M. Simonett, Jie Wang, et al., "Evaluation of automatically quantified foveal avascular zone metrics for diagnosis of diabetic retinopathy using optical coherence tomography angiography," Investigative Ophthalmology \& Visual Science, vol. 59, no. 6, pp. 2212-2221, 2018.

[7] Mingchao Li, Yuexuan Wang, Zexuan Ji, et al., "Fast and robust fovea detection framework for OCT images based on foveal avascular zone segmentation," OSA Continuum, vol. 3, no. 3, pp. 528, 2020.

[8] Menglin Guo, Mei Zhao, Allen M. Y. Cheong, et al., "Automatic quantification of superficial foveal avascular zone in optical coherence tomography angiography implemented with deep learning," Visual Computing for Industry, Biomedicine, and Art, vol. 2, no. 1, pp. 21, 2019.
[9] Morgan Heisler, Forson Chan, Zaid Mammo, et al., "Deep learning vessel segmentation and quantification of the foveal avascular zone using commercial and prototype oct-a platforms," arXiv:0707.3168 [eess], 2019.

[10] D. Karimi and S. E. Salcudean, "Reducing the hausdorff distance in medical image segmentation with convolutional neural networks," IEEE Transactions on Medical Imaging, vol. 39, no. 2, pp. 499-513, 2020 . 